\title{
Usage of a Web-Based Platform for Home Care Providers in the Pandemic Context
}

\author{
Virginia SANDULESCU ${ }^{\mathrm{a}, 1}$ Sorin PUSCOCI ${ }^{\mathrm{a}}$, Monica PETRE ${ }^{\mathrm{a}}$, Minodora \\ DUMITRACHE $^{\mathrm{a}}$, Alexandru GIRLEA ${ }^{\mathrm{b}}$ and Viorel BOTA ${ }^{\mathrm{c}}$ \\ ${ }^{a}$ National Institute for Studies and Research in Communications \\ b QuickWeb Info SRL \\ ${ }^{\mathrm{c}}$ Elisa Med SRL
}

\begin{abstract}
The paper presents the usage of a platform for home care providers that integrates telemonitoring functionalities in the context of the recent COVID-19 pandemic.
\end{abstract}

Keywords. remote health care, home care, tele-assistance

\section{Introduction}

Past year, in the COVID-19 pandemic context, we have faced a unique situation that urged the need of remote health provision. Telemonitoring offers the possibility to manage multiple subjects while minimizing human contact and the risk of spreading the SARS-COV-2 virus. [1] recommends telemedicine for monitoring different categories of COVID-19 patients or suspects. Through telemonitoring, relevant vital functions of suspected of confirmed COVID-19 patients may be effectively monitored so that symptoms exacerbations may be timely detected or even predicted and the number of hospital visits and admissions may be reduced [2], [3], [4].

The presented platform, called CDMS aims to optimize the activities of home care providers (HCPs). The current work focuses on the telemonitoring components of the platform: a web application for an HCP and APIs for integrating data from third party devices. More detailed descriptions about the platform are available in [5] and [6].

\section{Methods}

The CDMS platform facilitates offering health or social care services either through home visits or remotely: through videoconferences, messages or by using devices that may synchronize data over Internet.

The tele-assistance module presents the following functionalities for management of: patients; tele-assistance devices (environmental devices and medical devices); remote consultations through video conferences; tele-assistance data and tele-assistance alarms. The following capabilities of the platform facilitate remote health care: scheduling of

${ }^{1}$ Virginia Sandulescu, Department of Telematics and Communication Terminals, INSCC, Preciziei, 6, Bucharest, Romania; E-mail: virginia.sandulescu@inscc.ro. 
video consultations; communication with the patients through messages; upload medical documents for patients; consult and manage medical data for patients. Two APIs allow data synchronization from 3rd party environmental and health devices. The API for health data has been used to integrate the Mintti Vision 6in1 health monitoring device [7] for measuring blood pressure, heart rate, body temperature, oxygen saturation in arterial blood, glucose level and for performing ECGs. The measured values are automatically integrated in the HCP web app. Health data may also be entered manually by authorized medical personnel or, directly, by patients.

\section{Results, discussions and conclusions}

The CDMS platform is currently running as a pilot program in Alba County, Romania. It manages 3 HCPs via subscriptions. The largest of the three HCPs has 203 (at the time or writing) subscribed patients, receiving health care at home, both through home visits and remotely, or at a clinic.

The Mintti Vision 6in1 device has been successfully used to measure vital functions, including body temperature and $\mathrm{SpO} 2$, parameters that need to be monitored in case of COVID-19 infection.

At the moment, remote health care is preferred to face to face interactions and home visits are preferred to clinic visits in order to limit possible exposure to the Sars-Cov2 virus. The described tools facilitate video consults, online messaging, online medical recommendations, remote health monitoring, including remote monitoring of the health parameters that need to be measured in relation to the current threat.

\section{Acknowledgment}

This work has been supported through the Project: Dispatching and Management Center for Optimizing Home Care Integrated Services, from Operational Competitiveness Program 2014-2020 Axis 1 - Romanian Ministry of Research, Innovation and Digitalization.

\section{References}

[1] World Health Organization. Clinical management of severe acute respiratory infection when COVID-19 is suspected. 2020 .

[2] Ohannessian R, Duong TA, Odone A. Global telemedicine implementation and integration within health systems to fight the COVID-19 pandemic: A call to action. In: JMIR Public Health Surveill. 2020 Apr 2;6(2):e18810.

[3] Watson AR, Wah R, Thamman R. The value of remote monitoring for the COVID-19 pandemic. In: Telemedicine and e-Health. Sep 2020;1110-1112.

[4] Shaw JG, Sankineni S, Olaleye CA, Johnson KL, Locke JL, Patino J, Sabi FL, McCarthy RJ. A novel large scale integrated telemonitoring program for COVID-19. In: Telemedicine and e-Health. 2020.

[5] Sandulescu V, Puscoci S, Petre M, Soviany S, Girlea A, Bota V. Design and development of an integrated platform for management of homecare providers. In: E-Health and Bioengineering Conference (EHB). 2019;1-4.

[6] Sandulescu V, Puscoci S, Petre M, Girlea A, Bota V. Web application for home care providers. In: Stud Health Technol Inform. 2020 Jun 16;270:723-727.

[7] Mintti Vision, Product presentation, Mintti Health, Available at: http://minttihealth.com/?product=mintti-vision, Accessed on Jan. 11, 2021. 